\title{
O TEMPO COMO LEGITIMADOR DA CAUSA: IMPLICAÇÕES TEMPORAIS EM PESQUISAS DE ADMINISTRAÇÃO
}

TIME AS A LEGITIMATOR OF THE CAUSE: TEMPORAL IMPLICATIONS FOR RESEARCH IN BUSINESS ADMINISTRATION

EL TIEMPO COMO LEGITIMADOR DE LA CAUSA: IMPLICACIONES TEMPORALES EN INVESTIGACIONES DE ADMINISTRACIÓN

\author{
JEFERSON LANA \\ Doutor \\ Universidade do Vale do Itajaí - Brasil \\ jlana@univali.br \\ MARINA AMADO BAHIA GAMA \\ Doutora \\ Fundação Getúlio Vargas - Brasil \\ abgama@gmail.com \\ RODRIGO BANDEIRA-DE-MELLO \\ Pós-Doutor \\ Merrimack College - EUA \\ bandeiraderm@merrimack.edu \\ ROSILENE MARCON \\ Doutora \\ Universidade do Vale do Itajaí - Brasil \\ rmarcon@univali.br \\ Submetido: 15/12/2017 \\ Aprovado: 25/06/2018
}

Doi: alcance.v25n1(Jan/Abr).p106-119

\section{RESUMO}

Este artigo discute a importância do tempo nas pesquisas em administração de empresas e como a consideração dos efeitos temporais pode trazer novas abordagens de pesquisa. A ideia central do artigo é a de que o tempo legitima a causa, e que compreensão do tempo sobre a causa legitima a estratégia. Pesquisas causais em administração são mais facilmente absorvidas pela esfera corporativa, uma vez que servem como referências para o processo de tomada de decisão empresarial. Com as evidências encontradas em uma pesquisa bibliométrica sobre os estudos de efeitos temporais no Brasil, sugere-se que os pesquisadores devem se atentar aos efeitos temporais ainda na discussão teórica dos mecanismos e dos efeitos analisados. Dessa forma, espera-se que este artigo ajude no entendimento da importância do tempo nas pesquisas em administração e que sirva como motivação para estudos que busquem entender os fenômenos administrativos sob um ponto de vista diferente: dos efeitos temporais.

Palavras-Chave: tempo; efeitos temporais; causalidade; pesquisa em administração. 


\begin{abstract}
This article discusses the importance of time in research on business administration, and how the consideration of temporal effects can bring new research approaches. The central idea of the article is that time legitimates the cause, and that an understanding of time over the cause legitimates the strategy. Causal studies in business administration are more easily absorbed by the corporate sphere, as they serve as reference for the process of business decision-making. Based on evidence found in a bibliometric research of studies of temporal effects in Brazil, it is suggested that researchers pay attention to temporal effects in the theoretical discussion of the mechanisms of the effects analyzed. Thus, it is hoped that this article will further understanding of the importance of time in research in business administration, and that it will service to motivate studies that seek to understand the administrative phenomena from a different perspective; that of temporal effects.
\end{abstract}

Keywords: Time; Temporal effects; Causality; Research in business administration.

\title{
RESUMEN
}

Este artículo discute la importancia del tiempo en las investigaciones en administración de empresas y como la consideración de los efectos temporales puede traer nuevos abordajes de investigación. La idea central del artículo es la de que el tiempo legitima la causa, y que comprensión del tiempo sobre la causa legitima la estrategia. Investigaciones causales en administración son más fácilmente absorbidas por la esfera corporativa, una vez que sirven como referencias para el proceso de tomada de decisión empresarial. Con las evidencias encontradas en una investigación bibliométrica sobre los estudios de efectos temporales en Brasil, se sugiere que los investigadores se deben atentar a los efectos temporales aún en la discusión teórica de los mecanismos y de los efectos analizados. De esta forma, se espera que este artículo ayude en el entendimiento de la importancia del tiempo en las investigaciones en administración y que sirva como motivación para estudios que busquen entender los fenómenos administrativos sobre un punto de vista diferente: de los efectos temporales.

Palabras clave: Tiempo; Efectos temporales; Causalidad; Investigación en administración.

\section{INTRODUÇÃO}

Este artigo tem como objetivo ressaltar a importância do efeito do tempo em estudos provenientes das ciências sociais, com foco nas pesquisas de administração de empresas. Mais que um controle econométrico, compreender os efeitos temporais do tratamento entre as variáveis de interesse é um exercício teórico e que demanda um profundo conhecimento sobre os mecanismos que levam uma variável $X$ a influenciar uma variável dependente $Y$. O papel do tempo nessas relações não é trivial. $O$ tempo legitima a causa. $E$ a compreensão do tempo sobre a causa legitima a estratégia. Por exemplo, incentivos financeiros podem aumentar a produtividade dos funcionários, mas em quanto tempo? E por quanto tempo? Incubadoras e aceleradoras podem alavancar processos de inovação, mas quanto tempo leva para que uma empresa alcance bons resultados inovativos? Este artigo parte do princípio de que, ao compreender os efeitos temporais dos fenômenos administrativos, torna-se mais tangivel também avaliar as inferências causais sobre as relações pesquisadas.

A compreensão dos efeitos do tempo nas pesquisas de administração permanece uma questão aberta, principalmente nas discussões teóricas sobre as relações propostas. Enquanto grande parte da causalidade dos modelos econométricos utilizados em administração foca na seleção aleatória e desenhos metodológicos que controlem a endogeneidade das variáveis, pouca atenção se dá ao tempo de tratamento necessário para gerar esses efeitos. Neste artigo, trata-se este ponto como sendo crucial para o avanço das contribuições das pesquisas em administração. Apelos similares foram feitos na literatura da área e podem ser encontrados no estudo de Mitchell e James (2001) e Ancona, Goodman, Lawrence e Tushman (2001) sobre como construir melhores teorias, tendo em vista a exploração dos efeitos temporais, em uma edição publicado pela Academy of Management Review (AMR) ou ainda no livro de Kelly e McGrath (1988), o qual explora as relações entre tempo e métodos.

Ainda que pesquisas do tipo exploratórias, descritivas e associativas tenham sua importância inquestionável dentro do ambiente acadêmico administrativo, pesquisas causais permitem que 0 avanço teórico nos âmbitos acadêmicos e corporativos seja mais direto e impactante. Entender a relevância da causalidade é essencial para se compreender a importância do tempo nas relações estudadas. Causalidade é um dos aspectos 
científicos mais importantes, pois permite que o fenômeno seja mais bem compreendido e controlado (Roe, 2008; Angrist \& Pischke, 2015). Além disso, pesquisas causais em administração são mais facilmente absorvidas pela esfera corporativa, uma vez que servem como referências para o processo de tomada de decisão empresarial. No entanto, se o processo de inferir causa em ciências sociais não é tarefa simples, fazê-lo em administração parece ser ainda mais complicado. Não pela falta de esforço e conhecimento dos pesquisadores, mas pela essência dos fenômenos organizacionais, maturidade da área, acesso às informações, entre outros. Importante também ressaltar que pesquisas longitudinais são comuns à área de administração, mas o foco desses estudos é frequentemente 0 de testar efeitos ao longo do tempo, e não em se debruçar sobre os aspectos temporais que influenciam sua existência.

Processos e decisões organizacionais são gerados por numerosos motivos que agem paralelamente ou mediante interações. Esses motivos podem ser, por natureza, não observáveis, podem ter sua origem em fenômenos comportamentais e são, quase sempre, endógenos. Os esforços que têm sido feitos para melhorar esta realidade parecem focar mais nos aspectos metodológicos, principalmente quantitativos. Bons desenhos metodológicos são obviamente vitais para pesquisas causais, mas os aspectos teóricos não podem ser abandonados, pois são eles que justificam e norteiam os métodos. Antes dos procedimentos estatísticos, diversos exercícios teóricos precisam ser levados em conta. Compreender o papel do tempo é um desses exercícios, cujo resultado empírico torna os achados mais válidos e relevantes.

Neste artigo, aborda-se o fator tempo pela lente teórica das relações propostas nos estudos em administração de empresas e como sua análise determina as escolhas metodológicas e os procedimentos de interpretação. Portanto, o ponto de partida é a noção de que, assim como em qualquer campo de estudo científico, por exemplo, Biologia ou Educação, o tempo influencia as relações de interesse, do mesmo modo como o faz no campo organizacional. Um segundo ponto é que, embora este tema não seja novidade nos antecedentes teóricos (Mitchell \& James, 2001), a relevância do tempo em pesquisas de administração continua a ser uma questão em aberto, uma vez que sua discussão caminha a passos lentos e muitas vezes não suficientes. 0 terceiro ponto é que os pesquisadores devem se atentar aos efeitos temporais ainda na discussão teórica dos mecanismos e dos efeitos analisados, pois entender estes efeitos é mais importante que apenas testá-los empiricamente, além de potencializar inferências causais. $O$ quarto ponto é que a correta consideração do tempo nas discussões teóricas das relações de interesse levará a melhores decisões metodológicas. Por fim, o quinto e último ponto visa discutir as implicações metodológicas e interpretativas da inclusão do tempo no debate acadêmico em administração. Para evidenciar o estado da arte sobre as investigações de efeitos temporais em administração no Brasil, foi feita uma pesquisa bibliométrica com cinco dos principais periódicos brasileiros da área nos últimos seis anos. Foram encontrados 995 artigos publicados nestas revistas no período de 2010 a 2016, dos quais $56(5,26 \%)$ abordam 0 tema tempo empiricamente e apenas $12(1,21 \%)$ de forma empírico-teórica.

O público-alvo deste artigo se define por estudantes e pesquisadores de administração de empresas e áreas correlatas, como ciências contábeis, turismo, educação e psicologia. As contribuições aqui propostas se apoiam no entendimento de que as implicações temporais são relevantes ao avanço da compreensão dos mecanismos teóricos que explicam as relações entre variáveis. As contribuições ainda se estendem às discussões metodológicas de diferentes paradigmas. Discutir tempo e causalidade não é exclusividade de pesquisas quantitativas. Por exemplo, Widlok (2014) propõe análises de tempo e causa em investigação etnográfica. Dessa forma, espera-se que este artigo ajude no entendimento da importância do tempo nas relações organizacionais, principalmente causais, e que sirva como motivação para estudos que busquem entender os fenômenos administrativos sob um novo ponto de vista: 0 dos efeitos temporais.

\section{TEMPO: PASSADO, PRESENTE E FUTURO}

O conceito de tempo pode ser desafiador para determinadas áreas como é o caso da física. Ao buscar um conceito simplificado, Einstein definiu da seguinte forma: tempo é o que o relógio mede (Fischer, 1973). Importante para os estudos administrativos é a declaração de Benjamin Franklin: tempo é dinheiro. Neste artigo a ideia de tempo é entendida como o intervalo decorrido entre dois eventos a serem medidos. Esses eventos podem ser de qualquer natureza. Aqui, restringir-se-á aos eventos organizacionais, salvo exceções a título de exemplificação. Doravante, ao citar expressões como pesquisas ou pesquisadores, entenda-se que são aqueles ligados às pesquisas em Administração de Empresas, foco deste artigo. A seguir, apresenta-se a evolução dos estudos sobre efeitos temporais, sua interação com causalidade e suas perspectivas.

Revista Alcance - Eletrônica - vol. 25 - n. 1 - Jan./Abr. 2018 
Embora sejam parte das ciências sociais, os fenômenos organizacionais são estudados por uma série de teorias, algumas vezes concorrentes, que são escolhidas muitas vezes de forma subjetiva pelos pesquisadores. Ancona e colaboradores (2001) comentam que um fenômeno pode ser analisado pelas mais diferentes lentes teóricas e que cada uma destas lentes é capaz de revelar aspectos de interesse, enquanto negligenciam outros. É o custo de oportunidade também presente no ambiente acadêmico. Assim, quanto maior for a diversidade das visões com as quais se olha para um fenômeno, maior serão o número e a profundidade de explicações para ele. Ainda assim, algumas são mais utilizadas do que outras, e essa assimetria pode limitar a evolução do conhecimento. Dentre essas lentes que precisam maior atenção, pode-se citar o tempo. Não o tempo em si, em um conceito abstrato, mas o tempo como objeto ou variável que afeta as relações propostas pelos estudos organizacionais, sua magnitude e duração. Essa compreensão é essencial para o avanço teórico das pesquisas. É uma mudança da narrativa de pesquisas em administração, que migra de: "Quanto $X$ afeta $Y$ " para: "Quanto tempo é necessário para que $X$ afete $Y$ " ou "Quanto tempo duram os efeitos de $X$ em $Y$ ? ". Importantes periódicos da área, como o Academy of Management Review em 2001, já lançaram chamadas especiais sobre 0 tema. 0 mesmo já aconteceu para periódicos de psicologia, caso do Frontiers of Psychology, em 2014.

Além dos benefícios teóricos de compreender os efeitos temporais das relações organizacionais, a inclusão do tempo nos modelos tende a potencializar as inferências causais. Essa ideia já foi explorada há mais de 80 anos por Hall (1934), mas seus argumentos não poderiam ser mais atuais. Conforme cita Buehner (2014), tempo e causalidade estão firmemente presentes nas agendas de pesquisas sociais, ainda que o número de artigos que se aventurem por estes temas seja mais frequente em áreas como psicologia, educação e economia. A importância do tempo na causa se dá pelo conceito da direção temporal, que é frequentemente irreversível por sua essência em organizações. Conforme Hall (1934) já tratava, por exemplo, é possível juntar partículas de hidrogênio e oxigênio para formar água e, facilmente, reverter o processo, pois isso é parte de sua forma funcional. Já no caso entre fecundação e nascimento de bebês, obviamente há uma ordem irreversível de processos: um óvulo fecundado gera um bebê que, uma vez formado, não pode voltar a sua forma inicial. O mesmo ocorre com grande parte das estratégias empresariais, gestão de recursos humanos e demais vertentes da administração. As empresas podem abandonar uma ideia e cessar a fonte da causa, mas aquilo que foi gerado não será retornado em forma de recursos iniciais (George \& Jones, 2000). A causa precisa anteceder o efeito e, com frequência, este é um processo irreversível. Por isso, ao sugerir pesquisas organizacionais com inferências causais, os pesquisadores precisam fazê-lo tendo em vista que a causa deve sempre preceder ao efeito.

Mesmo que a lógica da direção temporal (em que a causa antecede o efeito por questões de irreversibilidade) pareça trivial, sua operacionalização traz grandes desafios aos estudos. Mitchell e James (2001) citam que é comum que as pesquisas deixem os intervalos entre causa e efeito não especificados, o que prejudica a interpretação dos resultados. Timmons e Preacher (2015) apontam que isso é uma consequência da falta de uma correta atenção aos modelos e aos desenhos de pesquisa, enquanto George e Jones (2000), Collins e Graham (2002) e Collins (2006) sugerem que, além de um problema metodológico, essa é principalmente uma questão teórica. Alguns exemplos de pesquisas organizacionais que levaram em consideração os efeitos temporais entre as variáveis de interesse podem ser encontrados em: Lee, Smith, Grimm e Schomburg (2000) para novos produtos e inovação; Schildt, Keil e Maula (2012) sobre aprendizagem organizacional; e Bridoux, Smith e Grimm (2013) sobre os efeitos temporais de diferentes ações sobre produtos e arena regulatória no desempenho da firma.

$O$ efeito temporal entre duas variáveis $X \rightarrow Y$ em que a seta indica que $X$ causa $Y$ pode ser estudado de diversas formas: ciclos, variáveis defasadas, duração, variações temporais, entre outros (Roe, 2008). A natureza do efeito temporal pode variar de acordo com a natureza das próprias variáveis e, com isso, as mudanças e os efeitos assumem formas diversas (Ployhart \& Vandenberg, 2010). A Figura 1 apresenta algumas dessas formas. Existem 1a) as formas lineares, em que 0 aumento do tempo leva a alterações direta ou indiretamente proporcionais do efeito; $1 \mathrm{~b}$ ) as formas não lineares, em que o tempo pode influenciar as mudanças de forma exponencial; e 1c) as mudanças que assumem forma descontínua por alguma alteração drástica sobre algum nível específico de tempo. 
Figura 1: Exemplos de Diferentes Formas de Relações e Mudanças

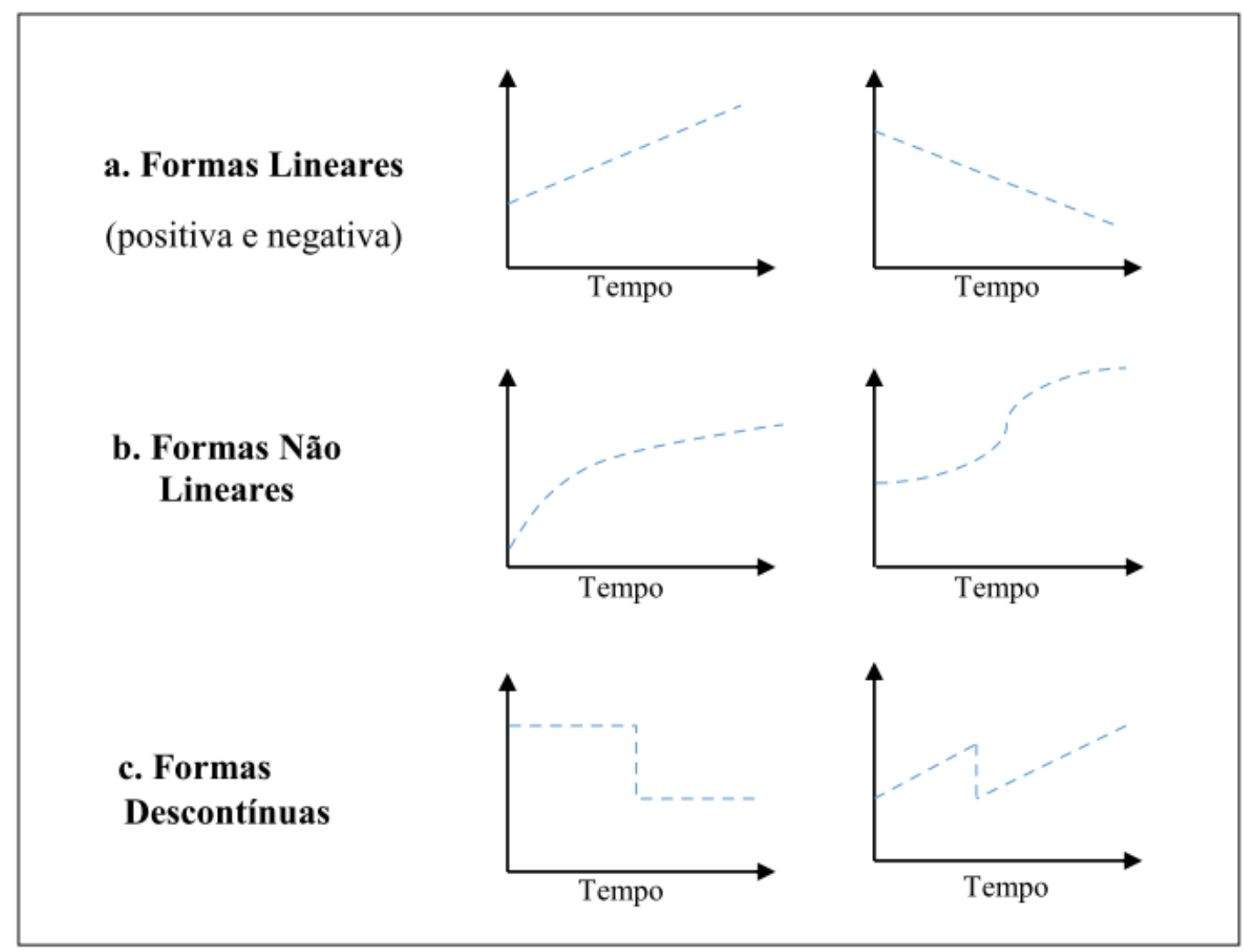

Fonte: Adaptada de Ployhart e Vandenberg (2010)

Embora Ployhart e Vandenberg (2010) utilizem essas diferentes formas da Figura $1 \mathrm{com}$ foco em mudanças e não especificamente em efeitos causais, a intuição por trás de ambos os raciocínios é igual. Assim, como exemplo de formas lineares (Figura 1, ponto a), pode-se citar a depreciação contábil de um galpão. Ainda que um investimento imobilizado possa trazer benefícios às empresas e seu valor contábil decresça linearmente no tempo. Já as formas não lineares (Figura 1, ponto b) são mais comuns. Por exemplo, empresas que oferecem incentivos sobre a produtividade observam, em geral, maior dedicação dos funcionários, mas em casos extremos 0 excesso de horas trabalhadas e o ritmo acelerado podem levar, inclusive, a quedas de produtividade no futuro após ultrapassar o nível ótimo da função. Por fim, formas descontínuas (Figura 1, ponto c) se caracterizam por mudanças bruscas. É o caso, por exemplo em finanças, do efeito de um rebaixamento da classificação do risco de uma empresa sobre seu custo de capital. Em 2014 e 2015, tal rebaixamento aconteceu com diversas empresas brasileiras pelas agências de rating. Uma vez que essas empresas perdem suas notas de classificação de risco, os bancos e demais instituições financeiras imediatamente passam a cobrar mais caro pelo capital. Assim, uma empresa que, por anos, reestruturou seu endividamento para reduzir o custo médio ponderado de capital pode de uma hora para outra observar uma disparada nos preços dos empréstimos. Por fim, é importante ressaltar que 0 acesso limitado às informações pode gerar uma interpretação errada ou incompleta do fenômeno (Ployhart \& Vandenberg, 2010). Pode ser o caso, por exemplo, de uma interpretação equivocada de linearidade decorrente de número limitado de observações representativas do fenômeno. Equívocos como este podem comprometer a validade e a contribuição de todo o estudo.

A análise dos efeitos temporais está intimamente ligada às pesquisas causais, mas não se restringe a elas. Pesquisas descritivas, exploratórias e correlacionais são igualmente beneficiadas ao adicionarem essa diferente lente sobre os fenômenos. Inclusive, não são raras as pesquisas causais que derivam de achados exploratórios ou descritivos, conforme citado por Hambrick (2007), que questionou o modelo vigente de artigos causais e com contribuições teóricas significativas para a área. George e Jones (2000) apontam que as análises temporais não precisam ser sempre causais. É importante, segundo os autores, que as pesquisas se atentem para o efeito temporal independente da classificação de suas contribuições. A seguir, são sugeridos alguns aspectos e questionamentos que um pesquisador pode fazer ao delimitar suas variáveis de estudo. 


\section{IMPLICAÇÕES TEÓRICAS DO EFEITO TEMPORAL}

O desenvolvimento teórico de uma hipótese deve ser capaz de explicar os mecanismos pelos quais a relação entre $\mathrm{Xe} Y$ acontece. Esses mecanismos devem estar presentes em estudos relacionais e, principalmente, causais. Por exemplo, indicar que grupos empresariais são característicos de países emergentes pode ser útil, mas ainda mais importante é a noção de que esses grupos são mais frequentes em países emergentes, uma vez que essa é uma forma de lidar com o ambiente institucional fraco e otimizar a gestão dos custos de transação (Khanna \& Yafeh, 2007). Em um artigo que explica o que realmente pode ser considerado uma contribuição teórica para o campo de administração, Sutton e Staw (1995) apontam que as hipóteses devem contar a história pela qual o fenômeno acontece, preferencialmente de forma causal, identificando qual variável vem antes e quando a relação é mais propensa a acontecer. Essa diferença sutil na forma de lidar com as hipóteses determina a contribuição do estudo e os avanços teóricos feitos pelos pesquisadores. É essa mesma intuição racional que se deve ter em mente para a análise dos efeitos temporais. 0 apelo principal deste artigo não é 0 de que os pesquisadores passem a avaliar unicamente os efeitos temporais dos fenômenos, mas sim que os levem em conta quando da determinação de uma relação (Mitchell \& James, 2001). Ployhart e Vandenberg (2010) citam um exemplo que pode ajudar a entender este raciocínio: em um estudo fictício de Recursos Humanos, em vez de formular uma hipótese dizendo que "comprometimento e intenção de rotatividade estão negativamente associados", o estudo geraria muito mais interesse se delimitasse a hipótese por "comprometimento e intenção de rotatividade possuem uma relação negativa que fica mais fraca com o passar do tempo". Ao adicionar aspectos temporais nas hipóteses, os autores as tornam mais falseáveis (Pitariu \& Ployhart, 2010).

Não existe um modelo padrão que determine como os efeitos temporais devam ser explorados. Cada relação teórica possui características únicas e cada área contém lacunas teóricas distintas que precisam ser preenchidas. Ainda assim, aqui é apresentado um modelo (Figura 2) com três momentos sequenciais de uma relação dita causal, os quais podem conter características temporais de interesse para diferentes estudos. Sua aplicação também pode servir em pesquisas correlacionais e exploratórias. Conforme mencionado anteriormente, o fato primordial para uma relação causal é que $X$ deva anteceder $Y$. Cada um dos momentos apresentados na Figura 2 são discutidos adiante.

Figura 2: Os processos da causalidade

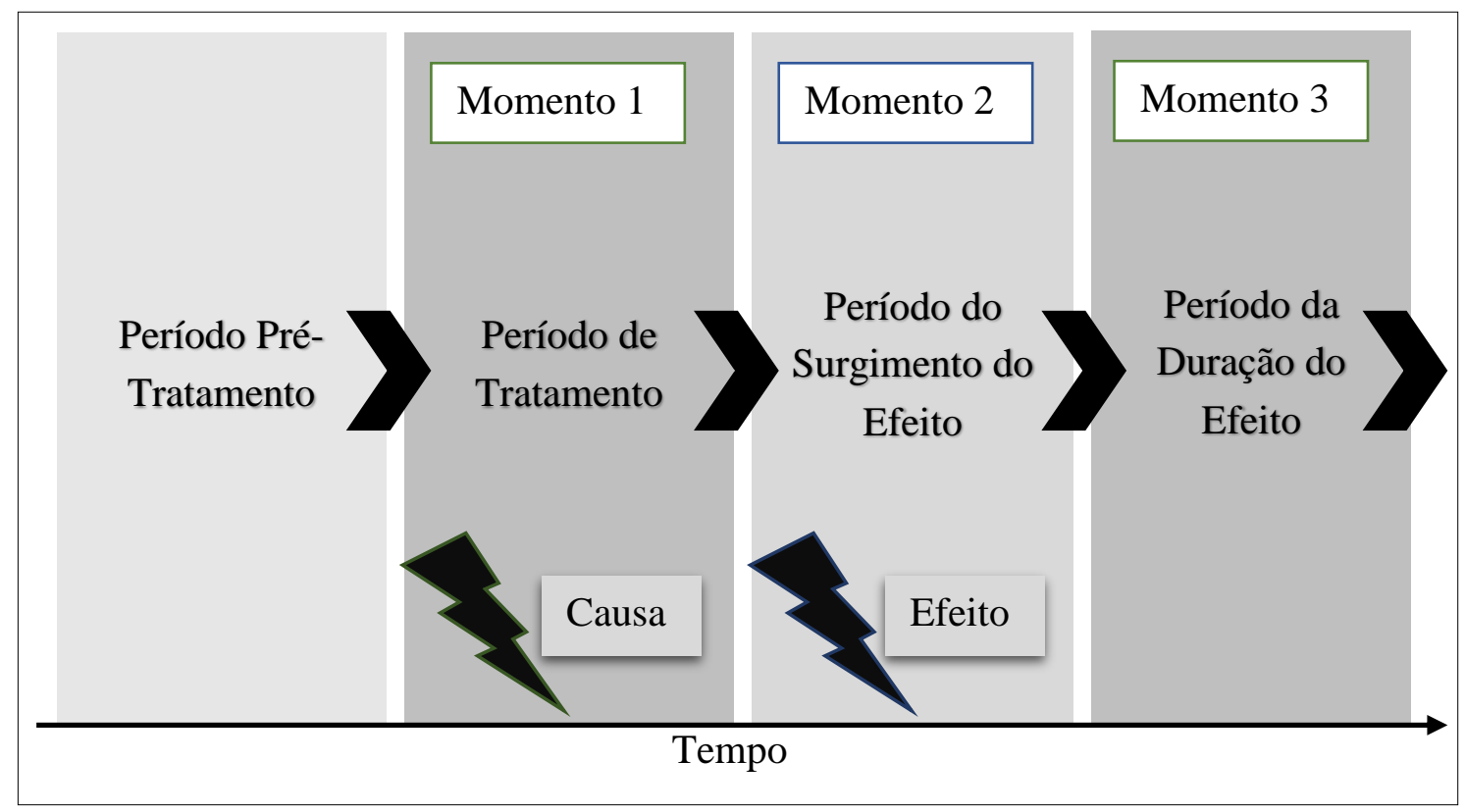

O modelo exposto na Figura 2 segue uma direção temporal e pode ser utilizada para compreender relações dos mais variados temas da administração, embora não se restrinja a ela. Para melhor compreender 0 modelo aqui exposto, optou-se por sua aplicação a um estudo real. Por exemplo, Hambrick, Misangyi e Park (2015) sugerem que a presença de um diretor de administração que condense quatro características específicas é um excelente preditivo da eficiência do conselho de administração, principalmente com foco no monitoramento. Dentre 
as quatro características, são citadas: independência, especialização na área da empresa, capacidade de comunicação e rede e motivação. 0 mecanismo causal citado pelos autores é que um profissional com tais características conseguiria mediar as discussões do conselho e encontrar meios para gerar soluções mais eficientes para o grupo. Estudos anteriores afirmavam que a eficiência do conselho de administração era determinada pela diversidade dos conselheiros; enquanto que a pesquisa de Hambrick, Misangyi e Park (2015) aponta que um fator importante é a presença de um único conselheiro que condense tal diversidade. Ao aplicar os momentos da Figura 2 ao estudo desses autores, espera-se que os conceitos de causalidade e efeitos temporais fiquem mais claros e sirvam como exemplo para demais estudos:

i. Período Pré-Tratamento: é o momento em que o objeto de estudo da pesquisa, seja ele um indivíduo, equipe ou empresa, ainda não foi tratado por uma variável $X$ qualquer. É importante também que ainda não haja a intenção de tratá-la, pois isso poderia gerar endogeneidade no efeito causal e viés de seleção sobre as empresas tratadas. No exemplo citado anteriormente, é quando as empresas desenvolvem suas atividades sem ter esse "superconselheiro". Nesta parte do processo, alguns artigos preferem focar no conceito de timing em vez de tempo, pois essa escolha ajudaria a entender quando uma empresa está mais sujeita a receber determinado tratamento. O estudo de Bonardi, Hillman e Keim (2005) é um exemplo que ajuda a entender o conceito de timing em estratégias políticas corporativas pela ideia de mercados políticos.

ii. Momento 1 - Período de tratamento: 0 período de tratamento é entendido como 0 intervalo necessário para a implantação de X. Em alguns casos, o período de tratamento pode ser extremamente curto, como o exemplo citado anteriormente sobre o impacto do rebaixamento da nota de classificação de risco de uma empresa sobre seu custo de capital. Como os contratos financeiros são indexados a estas notas, uma alteração de classificação gera um imediato aumento de custos. De particular interesse aqui é o tempo que $X$ leva para ocorrer, que neste caso é imediato, pois representa o anúncio da nova nota pela agência de risco ao mercado. Em outros casos, o período de tratamento é mais longo. $O$ treinamento de funcionários pode ser citado como um desses casos, principalmente quando o período de realização é longo. Assim, para um treinamento fictício de 10 dias (tempo $t$ ), espera-se que 0 intervalo entre as mensurações de $X$ e $Y$ seja de, ao menos, 11 dias $(t+1)$, sem levar em consideração aspectos como a curva de aprendizagem. No estudo de Hambrick, Misangyi e Park (2015), o período de tratamento se considera pelo tempo necessário para que o novo conselheiro consiga implantar as ações necessárias para melhoria da eficiência de monitoramento. Esse intervalo pode ser de dias ou meses. Os autores não abordam esse tópico em profundidade. 0 período de tratamento é algo comumente negligenciado pelos antecedentes teóricos que costumam se interessar exclusivamente pelo tempo entre $\mathrm{X}$ e $\mathrm{Y}$ (Mitchell \& James, 2001). Em experimentos com seleção aleatória, os modelos empíricos costumam operacionalizar o tratamento de $\mathrm{X}$ como se ele fosse imediato. Com frequência ele não o é.

iii. Momento 2 - Período do Surgimento do Efeito: dentre as pesquisas sobre efeitos temporais, esta é a abordagem mais comum. $O$ período do surgimento do efeito é entendido como o intervalo de tempo decorrente entre a implantação de $\mathrm{X}$ e $\mathrm{o}$ seu efeito em $\mathrm{Y}$. Em outras palavras, quanto tempo depois de $\mathrm{X}$ ocorrerá $\mathrm{Y}$. No exemplo sugerido de Hambrick, Misangyi e Park (2015), seria o tempo entre a contração e a efetivação do conselheiro no cargo e as melhorias da eficiência de monitoramento. Um ponto importante de se observar aqui é que as empresas podem (e vão) apresentar intervalos de tempo diferentes entre si. Caso não haja evidência teórica que explique essa heterogeneidade dos intervalos ou o acesso às informações não seja possível, os pesquisadores podem utilizar intervalos médios. Angrist e Pischke (2015, p. viii) citam que: "'on average' is usually good enough". Com isso, é comum encontrar artigos ou teses que investiguem a implantação de X em um ano "t" nos efeitos de $Y$ (em " $t$ " ou mesmo em " $t+1$ "), sem questionar o porquê dessas definições ou apenas justificando que essa é uma prática comum na literatura da área. Essa escolha não está errada, principalmente se o foco do estudo não trata de aspectos temporais. As definições dos intervalos temporais e diferenças entre grupos devem ser guiadas pela lente teórica ou, caso não haja indicação, por pesquisas exploratórias. As relações entre $X$ e $Y$ podem assumir diferentes formas e configurações, como causalidade reversar, moderação por variáveis $Z$ ou formação de uma terceira variável $Q$ (para uma melhor compreensão destas formas funcionais de $X \rightarrow Z$, ver Mitchell e James, 2001). Outro ponto importante é definir se X muda ao longo do tempo. Ponderações como essas podem elevar o debate acadêmico.

iv. Momento 3 - Período da Duração do Efeito: o período da duração do efeito representa o intervalo que vai do surgimento do efeito até o ponto em que ele existir. Alguns efeitos podem ser considerados constantes, enquanto outros podem apresentar crescimento ou diminuição em suas magnitudes. É importante compreender que o tempo não explica os fatores que causam a variação dos efeitos no intervalo de tempo, mas ajuda a demonstrar como o efeito se comporta temporalmente. No caso do estudo de Hambrick, Misangyi e Park (2015),

Revista Alcance - Eletrônica - vol. 25 - n. 1 - Jan./Abr. 2018 
o período da duração do efeito da presença do conselheiro pode durar meses ou anos. Hillmann (2005) encontrou que a nomeação de conselheiros politicamente conectados gera benefícios significantes por até 2 anos após a nomeação. Pesquisas que focam na duração do efeito são de grande interesse para pesquisas ligadas a Recursos Humanos e Psicologia. Por ser subjetivo, aspectos como o comportamento humano tendem a variar consideravelmente ao longo do tempo e, por isso, recebem maior atenção por parte dos pesquisadores. Por exemplo, Boswel, Boudreau e Tichy (2005) apresentam o efeito da duração da satisfação de trabalho a partir das mudanças de trabalho. Pesquisas exploratórias e de associações também costumam ser feitas sob esta perspectiva.

Com isso, a Figura 2 demonstra que o processo de causalidade possui ao menos 3 momentos distintos que podem servir como base para o questionamento das hipóteses elaboradas para estudos de pesquisa em administração: "Quanto tempo demora para que X aconteça? ", "Em quanto tempo X gera efeitos em $Y$ ? " e, por fim, "Por quanto tempo os efeitos de X em $Y$ duram?". Essas perguntas podem ajudar os pesquisadores a definir melhor o escopo, mecanismos teóricos e compreensão dos fenômenos organizacionais que pretende investigar. Ajudam também nas definições metodológicas, conforme tratado a seguir.

\section{ASPECTOS METODOLÓGICOS DO EFEITO TEMPORAL}

Os aspectos metodológicos de uma pesquisa devem ser guiados pela natureza do fenômeno a ser estudado, pelos mecanismos teóricos por meio dos quais ele se explica e pelas hipóteses desenvolvidas, que devem favorecer sua própria falseabilidade. Com os efeitos temporais não é diferente. Mas de que forma o efeito temporal pode avançar no entendimento de um fenômeno? Para Timmons e Preacher (2015), pesquisas que não se atentam aos aspectos temporais podem gerar contribuições incompletas e distorcidas sobre como as relações se desenvolvem ao longo do tempo. Anteriormente neste artigo foram definidas algumas formas sobre como endereçar os efeitos temporais pela lente teórica e em quais momentos do processo de causalidade o tempo é fator relevante. Nesta seção, estas questões são endereçadas de forma empírica, com foco nos métodos quantitativos e sua operacionalização.

Um primeiro aspecto de interesse, segundo Roe (2008, p.40), é que os pesquisadores estão mais acostumados a pensarem em termos de "qual o efeito", em vez de "o que acontece quando". Ancona e outros (2001) sugerem que esse viés mental tem sua origem nas dificuldades em se estudar aspectos causais, dentre os quais cita: oportunismo do pesquisador (que tende a focar em número de produções, em vez de profundidade), convenções de pesquisa (aquelas com as quais está mais acostumado, como experimentos), falta de uma teoria que guie novas pesquisas temporais e, por fim, a falta de metodologias e experiência prática. Aqui se foca apenas na parte metodológica, buscando avançar no entendimento sobre como testar hipóteses temporais empiricamente. Assim, abrangem-se aspectos como a natureza das variáveis e das técnicas estatísticas que otimizem os resultados dos efeitos temporais.

Algo que precisa ficar claro é que pesquisas de efeitos temporais são relacionadas às pesquisas longitudinais, mas não são sinônimas (Timmons \& Preacher, 2015). Para entender o efeito temporal, o pesquisador precisará de dados longitudinais com ao menos dois períodos de observação para o mesmo indivíduo ou objeto de estudo. No entanto, nem todas as pesquisas longitudinais buscam entender o efeito temporal de um fenômeno. Estudos temporais podem focar em diversos aspectos: intervalos temporais, defasagem entre causa e efeito, duração, ciclos, ondas, entre outros (Collins, 2006), algumas das quais já foram contempladas na Figura 2. Independentemente da forma escolhida ou sugerida pela teoria, os aspectos metodológicos se dividem em três etapas, descritas a seguir. Estas etapas ajudam a melhor visualizar as opções metodológicas, embora não esgotem as opções pelas quais o tempo pode ser avaliado empiricamente.

A primeira etapa é definir pela teoria o intervalo de tempo capaz de medir o efeito entre as variáveis. Por exemplo, Mitchell e James (2001) citam uma configuração de relações em que X leva a $Y$ que leva a $Q(X \rightarrow Y \rightarrow Q)$. Logo, seguindo a lógica de que as causas antecedem os efeitos, essas variáveis não podem ser relacionadas no mesmo tempo $t$. Empiricamente, tem-se que $X$ deve estar em tempo $t$, $Y$ em " $t+1$ " e $Q$ em " $t+2$ " para que seja possivel testar as relações. Ainda assim, o tempo entre $X$ e $Y$ pode ser diferente entre $Y$ e $Q$, e esses aspectos precisam ser levados em consideração. Outra opção seria o uso das variáveis defasadas, em tempo "t-1", por exemplo. $O$ ponto crucial é que se o intervalo de tempo para que $\mathrm{X}$ gere um efeito em $\mathrm{Y}$, espera-se que a base de dados contenha observações semanais ou mensais. A unidade de medida da primeira etapa deve ser igual ou menor que o intervalo de medida da segunda etapa. 
Já a segunda etapa diz respeito aos intervalos de medida. Intervalos de medida podem ser entendidos como o espaço de tempo entre duas observações. Esses intervalos podem ser diários, mensais, trimestrais, etc. Cada área de administração possui intervalos mais comuns. Por exemplo, nas pesquisas em estratégia empresarial, o intervalo de medida tradicional é o anual. Ainda assim, o que deve nortear o intervalo de medida é a natureza das relações e não exclusivamente os períodos tradicionalmente utilizados. Os intervalos de medida são importantes para se verificar intervalos temporais de efeito e duração. Timmons e Preacher (2015) focam neste aspecto e oferecem um pacote específico para o software $R$, que ajuda a efetuar tais análises. Conforme os autores citam, uma regra de bolso para pesquisa é que intervalos cada vez menores são importantes, pois podem ajudar a entender a natureza das relações, facilitar o encontro de efeitos e aumentar a precisão dos testes. Ainda assim, amostras mais frequentes podem ter um alto custo de oportunidade e é preciso que essas decisões também sejam guiadas pela teoria. Pelos intervalos de medida mais frequentes é possível determinar casos, por exemplo, de não linearidade de efeitos (Figura 1), ciclos, duração dos efeitos, entre outros. Uma pesquisa com intervalo de medida anual, que busque entender o impacto do aumento salarial na motivação de um funcionário, pode não encontrar significância, ao passo que a mesma pesquisa com intervalos semanais ou mensais pode identificar esse efeito como tendo, por exemplo, 4 meses de duração. Conforme já mencionado, não há uma fórmula pronta para definir como os dados devem ser analisados. Cabe ao pesquisador, guiado pela teoria, fazer essas escolhas de forma a otimizar as inferências dos resultados.

Por fim, a terceira etapa trata das técnicas e dos métodos estatísticos a serem utilizados para os testes das relações propostas. Assim, são apresentadas algumas das escolhas estatísticas possíveis pelo pesquisador. Aqui a Figura 1 volta a ser importante, uma vez que os testes dependem não apenas dos intervalos de medição, mas principalmente da natureza das relações, se lineares, não lineares, etc. Variáveis de natureza lineares podem ser estudadas por meio de regressões lineares múltiplas convencionais, com adição de aspectos de efeitos fixos para controle de variáveis não observáveis ou ainda com coeficientes robustos que controlam pela heterocedasticidade. Também podem ser utilizadas técnicas estatísticas mais simples, como os estudos de evento ou análises de variância simples e multivariadas ou modelos hierárquicos lineares. Já para os casos de não linearidade em modelos exponenciais, cíclicos, entre outros, Mitchell e James (2001) citam a importância da utilização de técnicas, como os growth curve models. Cabe ressaltar que estas técnicas são apenas algumas dentre uma vasta gama possível de técnicas existentes. Para outros exemplos de técnicas causais, pode-se citar o livro de Angrist e Pischke (2015) ou Stanley e Campell (1963).

Assim, tendo o processo, finaliza-se com a seguinte ordem temporal pelo ponto de vista do pesquisador: primeiro surge a ideia do tema, guiada pela revisão da literatura ou pelo conhecimento em profundidade do assunto. Em seguida, sugerem-se algumas das hipóteses iniciais, cujas explicações e conteúdo foram guiados pelos mecanismos teóricos. Em seguida, ainda com o apoio da teoria, são definidos os aspectos como intervalo de efeito e intervalos temporais. Por fim, essas decisões e pilares teóricos ajudam a definir as técnicas empíricas que serão utilizadas para análise dos dados. Este não é um modelo de como devem funcionar as pesquisas em administração, mas essa sequência pode ajudar os pesquisadores a entenderem os passos do processo, cujo resultado final deva ser um estudo que gere contribuições práticas, empíricas e teóricas.

\section{O EFEITO TEMPORAL NO BRASIL}

Com o objetivo de ilustrar o estado da arte das pesquisas temporais em Ciências Sociais no Brasil, foi feito um breve levantamento bibliométrico entre cinco das principais revistas acadêmicas do País: Revista de Administração de Empresas (ERA, da Fundação Getúlio Vargas), Revista de Administração Contemporânea (RAC, da Associação Nacional de Pós-Graduação e Pesquisa em Administração, ANPAD), Business Administration Review (BAR, também da ANPAD), Revista de Administração da Universidade de São Paulo (RAUSP, da Universidade de São Paulo, USP), Revista Brasileira de Gestão e Negócios (RBGN, da Fundação Escola de Comércio Álvaro Penteado, FECAP). Esta lista foi selecionada a partir dos critérios de classificação e avaliação dos periódicos de 2015 desenvolvidos pela CAPES. O período da análise se deu entre 2010 a julho de 2016, com o intuito de avaliar um período mínimo de cinco anos, a contar da data do desenvolvimento deste artigo. A busca feita nestes periódicos se refere ao uso teórico (no desenvolvimento teórico das hipóteses ou das proposições) e ao uso empírico (nos testes e nos aspectos metodológicos) do tempo.

Para o período delimitado, de 2010 a 2016 (seis anos e seis meses), dentro do escopo dos cinco periódicos selecionados, foram encontrados um total de 995 artigos publicados. Este número representa uma média de 154 artigos por ano, ou cerca de 13 artigos por mês. Após o levantamento e o download de todos os 995

Revista Alcance - Eletrônica - vol. 25 - n. 1 - Jan./Abr. 2018 
artigos, por meio do site de cada uma das revistas, foi feita uma busca textual sobre termos relacionados ao tema de efeito temporal. Este procedimento foi feito a partir da utilização do software Atlas TI, para auxílio na codificação e na categorização dos dados da busca e análise do conteúdo. Na Tabela 1, é possível ver as palavras utilizadas para a codificação dos dados. Após a codificação de todos os artigos, foi feita uma análise minuciosa e individual para categorização dos dados encontrados. Esta categorização foi dividida em artigos cujos achados de efeitos temporais estavam no desenvolvimento teórico, chamado de "Tempo-Teórico" e aqueles artigos cujos achados estavam mais concentrados na parte empírica da pesquisa, aqui chamados de "Tempo-Empírico". Essa classificação se destina a encontrar aqueles artigos que não apenas testam efeitos empíricos temporais pelo uso, por exemplo, de variáveis defasadas ou retornos anormais no tempo, mas que também se prestam ao desenvolvimento e contribuição teóricos quanto à pesquisa de efeitos temporais. Em seguida, utilizou-se a ferramenta query tool do Atlas TI para fazer a análise, utilizando-se álgebra booleana, e para verificar quais artigos somente utilizavam o "Tempo-Empírico" e quais artigos utilizavam tanto o "Tempo-Teórico" e "Tempo-Empírico".

Dentro deste universo de 995 artigos publicados, apenas 56 (5,63\%) tratavam de aspectos temporais, seja pelas contribuições do desenvolvimento teórico ou pelas verificações empíricas. Quando as análises individuais dos 56 artigos restringiram os achados àqueles que se propõem a evoluir concomitantemente nas discussões temporais teóricas e empíricas, o número de artigos publicados encontrados totalizou $13(1,30 \%$ do universo investigado) casos. Estes 13 artigos tratavam o efeito temporal tanto na parte teórica quanto na parte empírica. Os resultados da análise são apresentados na Tabela 1.

Tabela 1: Palavras utilizadas na codificação dos artigos

\begin{tabular}{l|l}
\hline Categoria de Análise & Codificação \\
\hline Tempo-Teórico & Evento \\
\hline & Ciclo \\
\hline & Tempo \\
\hline & Time \\
\hline & Temporal \\
\hline Tempo-Empírico & Longitudinal \\
\hline & Longitudinal \\
\hline & Panel \\
\hline & Painel \\
\hline & Defasado (a) \\
\hline & Lagged, Lag \\
\hline & Evento \\
\hline & Event study
\end{tabular}


Figura 3 - Representação dos achados nos antecedentes teóricos

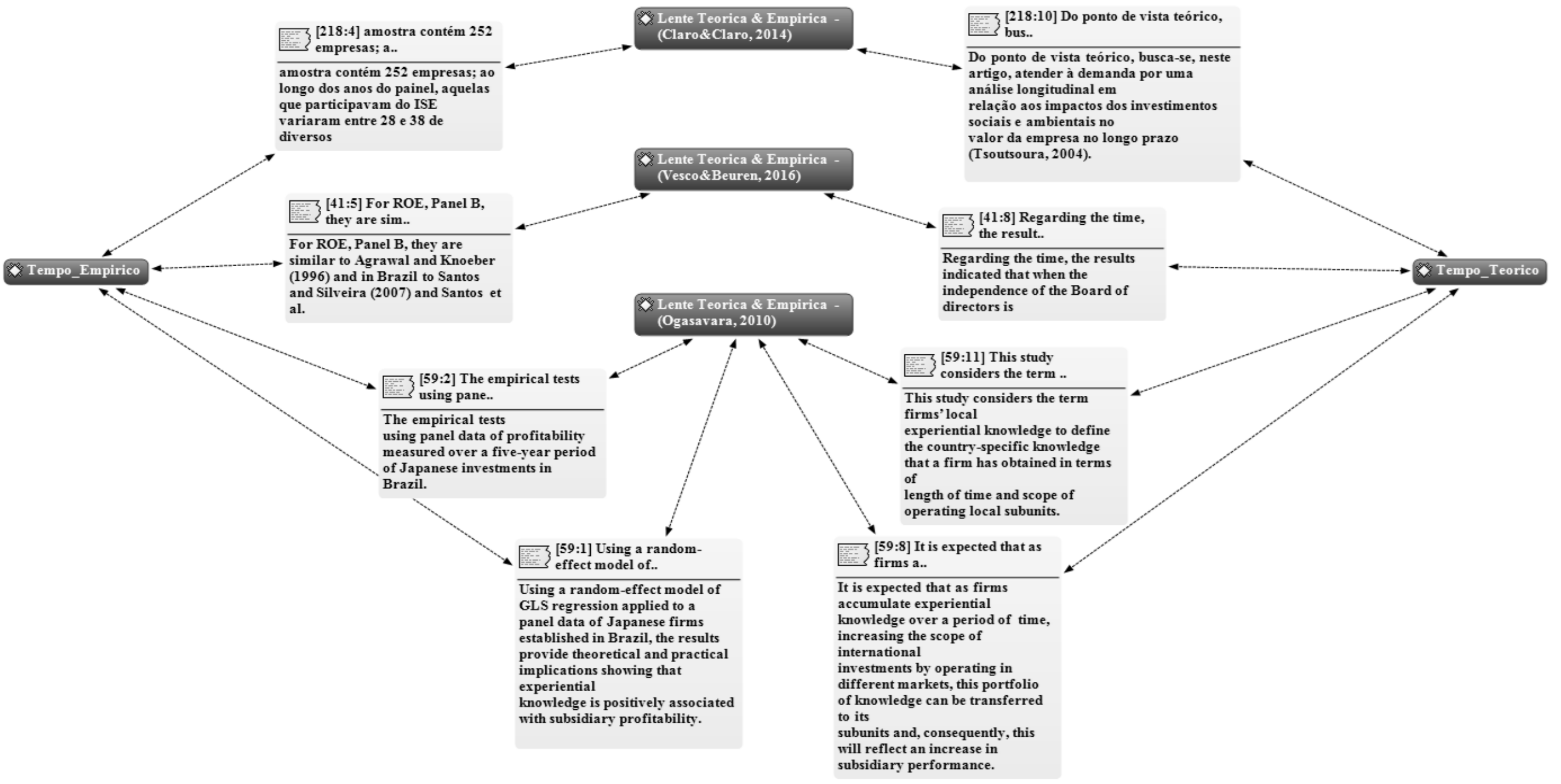


Este levantamento ajuda a elucidar a necessidade de que novos artigos passem a explorar o efeito temporal na teoria em conjunto com o efeito temporal empírico, de forma que justifiquem a utilização das técnicas estatísticas baseadas em tempo e que levem em consideração os efeitos temporais para melhorar as análises causais. Por exemplo, o estudo de Oliveira, Maçada e Oliveira (2015) justifica a importância do uso do tempo já nas hipóteses e, como consequência, faz uso de estudo longitudinal com dados em painel. Um outro exemplo, o artigo de Silva e Famá (2011), utiliza o estudo de evento como técnica estatística e também discute a questão do tempo e a relação com a precificação dos ativos ainda na parte teórica do estudo e depois testa as hipóteses com estudo de eventos que utiliza o tempo como principal mecanismo de teste. Lazzarini, Brito e Chadad (2013) exploram a vantagem competitiva ao longo do tempo e as alianças estratégicas, assim como utilizam dados em painel. No entanto, a grande maioria dos artigos analisados utilizam o efeito temporal apenas na parte empírica do estudo, como uso de dados em painel, estudos longitudinais e estudos de evento. $\mathrm{Na}$ Tabela 2, estão evidenciados os artigos que consideram e efeito temporal teórico como parte do desenvolvimento do artigo. Embora artigos que tratem os efeitos temporais de forma empírica ajudem a elucidar aspectos relevantes aos fenômenos investigados, a presença dos efeitos temporais também nas discussões teóricas ajuda a potencializar a relevância e a validade das contribuições sugeridas.

Tabela 2: Artigos que utilizam efeitos temporais pelo prisma teórico e empírico

\begin{tabular}{c|c|c}
\hline Artigo & Autor(es) / ano & Revista \\
\hline $\begin{array}{c}\text { Organizational Commitment, Psychological Contract Fulfillment and } \\
\text { Job Performance: A Longitudinal Quanti-qualitative Study }\end{array}$ & Maia e Bastos (2015) & BAR \\
\hline $\begin{array}{c}\text { Corwin-Schultz Bid-ask Spread Estimator in the Brazilian Stock } \\
\text { Market }\end{array}$ & Ripamonti (2016) & BAR \\
\hline $\begin{array}{c}\text { Do the Board of Directors Composition and the Board } \\
\text { Interlocking Influence on Performance? }\end{array}$ & Vesco e Beuren (2016) & BAR \\
\hline $\begin{array}{c}\text { Conduits of Innovation or Imitation? Assessing the Effect of Alliances } \\
\text { on the Persistence of Profits in U.S. Firms }\end{array}$ & Lazzarini, Brito, Chadad (2013) & BAR \\
\hline $\begin{array}{c}\text { The Role of Experiential Knowledge and Subsequent Investment } \\
\text { Decisions on the Profitability of Japanese Companies in Brazil }\end{array}$ & Ogasavara (2010) & BAR \\
\hline $\begin{array}{c}\text { Valor da Tecnologia da Informação na Firma: Estudo com Empresas } \\
\text { Brasileiras }\end{array}$ & Oliveira, Maçada e Oliveira (2015) & RAC \\
\hline $\begin{array}{c}\text { Tangibilidade e Intangibilidade na Determinação do Desempenho } \\
\text { Persistente de Firmas Brasileiras }\end{array}$ & Carvalho, Kayo, Martin (2010) & RAC \\
\hline $\begin{array}{c}\text { Análise do retorno dos investimentos socioambientais das empresas } \\
\text { brasileiras }\end{array}$ & Orlleano e Quiota (2011) & RAE \\
\hline $\begin{array}{c}\text { Key factors in working capital management in the Brazilian market } \\
\text { Corporate social responsibility And financial performance: the } \\
\text { Spanish case }\end{array}$ & Palombini e Nakamura (2012) & RAE \\
\hline $\begin{array}{c}\text { Cvidências de retornos anormais nos processos de IPO na Bovespa } \\
\text { no período de 2004 a 2007: um estudo de evento }\end{array}$ & Madorran e Garcia (2016) & RAE \\
\hline Sustentabilidade estratégica: existe retorno no longo prazo? & Claro e Claro (2014) & RAUSP \\
\hline $\begin{array}{c}\text { Maturidade do endividamento, desenvolvimento financeiro e } \\
\text { instituições legais: análise multinível em empresas latino- } \\
\text { americanas }\end{array}$ & Martins e Terra (2015) & RAUSP \\
\hline
\end{tabular}

\section{CONSIDERAÇÕES FINAIS}

Neste ensaio teórico focou-se a importância do tempo nos estudos de administração de empresas. Se bons estudos mostram novas formas de ver e entender o mundo, a lente dos efeitos temporais é, com certeza, uma dessas formas relevantes de enxergar os fenômenos. Ao se atentar para o tempo, o estudo pode não só medir o impacto das relações, mas o comportamento desses impactos ao longo de um intervalo. Conforme apresentado na primeira seção, a ideia de causalidade é importante para a geração de novas contribuições teóricas. Um estudo não precisa ser causal para ser um bom estudo. Mas pesquisas causais permitem que a comunidade se apoie em ideias e teorias mais validadas e com relativa capacidade de explicação. Foi visto também que o conceito de causalidade está intimamente ligado à ideia de irreversibilidade especialmente em estudos organizacionais. E é aí que está o link entre causa e tempo. Ao falar de causalidade, os pesquisadores 
precisam se ocupar dos efeitos temporais para explicar o comportamento desses efeitos. 0 tempo legitima a causa. A causa legitima as decisões estratégicas.

A segunda e a terceira seção buscaram tratar de forma mais prática e elucidativa as implicações teóricas e empíricas dos efeitos temporais. $O$ artigo buscou direcionar a luz para um ponto ainda pouco claro, mas relevante das teorias organizacionais e econômicas usadas no campo administrativo. Acredita-se que quanto maior for a atenção da área de administração aos efeitos temporais das relações estudadas, maiores serão as contribuições advindas. Os argumentos e os apelos se basearam em dois alicerces: na falta de profundidade da questão temporal observada por meio da análise de artigos brasileiros, orientações, bancas e avaliações de artigos. A outra fonte de conteúdo veio dos antecedentes teóricos que sugerem anseios e visões compartilhadas, principalmente aqueles presentes no estudo de Mitchell e James (2001), Roe (2008) e Ployhart e Vandenberg (2010).

Não há uma regra que indique a obrigação do uso dos efeitos temporais sobre os estudos organizacionais. Esta deve ser uma escolha particular do pesquisador e que faça sentido dentre do seu tema de pesquisa. No entanto, após uma vasta revisão bibliográfica, não foi encontrada uma só referência que indique que a profunda compreensão dos aspectos temporais de um fenômeno prejudique qualquer artigo.

\section{REFERÊNCIAS}

ANCONA, D. G., GOODMAN, P. S., LAWRENCE, B. S., TUSHMAN, M. L. Time: A new research lens. Academy of Manegement Review, v. 26, n. 4: p. 645-663, 2001.

ANGRIST, J. D., PISCHKE, J. S. Mastering Metrics: the path from cause to effect. Princeton, NJ: Princeton University Press, 2015.

BOSWELL, W. R., BOUDREAU, J. W., TICHY, J. The relationship between employee job change and jog satisfaction: the honeymoon-hangover effect. Journal of Applied Psychology, v. 90, p. 882-892, 2005.

BRIDOUX, F., SMITH, K. G., GRIMM, C. M. The management of resources: Temporal effects of different types of actions on Performance. Journal of Management, v. 39, n. 4, p. 928-957, 2013.

BUEHNER, M. J. Time and Causality: editorial. Frontiers in Psychology, v. 5, p. 1-2, 2014. Doi: 10.3389/spsyg.2014.00228.

CAMPBELL, D. T., STANLEY, J. C. Experimental and quasi-experimental designs for research. Chicago: Rand McNally, 1963.

CARVALHO, F. M.; KAYO, E. K.; MARTIN, D. M. L. 2010. Tangibilidade e Intangibilidade na Determinação do Desempenho Persistente de Firmas Brasileiras. Revista de Administração Contemporânea, v. 14, n. 5, p. 871 889, 2010.

CLARO, P. B. O.; CLARO, D. P. Sustentabilidade estratégica: existe retorno no longo prazo? RAUSP, v. 49, n. 2, p. 291-306, 2014.

COLLINS, L. M. Analysis of longitudinal data: The integration of theoretical model, temporal design, and statistical model. Annual Review of Psychology, v. 57, p. 505-528, 2006.

COLLINS, L. M.; GRAHAM, J. W. The effect of timing and spacing of observations in longitudinal studies of tobacco and other drug use: Temporal design considerations. Drug and Alcohol Dependence, v. 68, p. 85-86, 2002.

FISCHER, A. What time is it - really? Popular Science, v. 202, n. 3, p. 110-112, 1973.

GEORGE, J. M.; JONES, G. R. The role of time in theory and theory building. Journal of Management, v. 26, p. 657:684, 2000.

HALL, E. W. Time and Causality. The Philosophical Review, v. 43, n. 4, p. 330-350, 1934. Disponível em: $<$ http://www.jstor.org/stable/2180151>.

Hambrick, D. C. 2007. The Field of Management's Devotion to Theory: too much of a good thing? Academy of Management Journal, 50(6): 1346-1352.

HENRI, S.; KEIL, T.; MAULA, M. The temporal effects of relative and firm-level absorptive capacity on interorganizational learning. Strategic Management Journal, v. 33, p. 1154-1173, 2012. 
HILLMANN, A. J. Politicians on the Board of Directors: Do Connections Affect the Bottom Line? Journal of Management, v. 31, n. 3, p. 464-481, 2005.

KELLY, J. R.; MCGRATH, J. E. On time and method. Newbury Park, CA: Sage, 1988.

KHANNA, T.; YAFEH, Y. Business groups in emerging markets: Paragons or parasites? Journal of Economic Literature, v. 45, n. 2, p. 331-372, 2007. Disponível em: http://www.jstor.org/stable/10.2307/27646796.

LAZZARINI, S. G.; BRITO, L.; CHADAD, F. Conduits of Innovation or Imitation? Assessing the Effect of Alliances on the Persistence of Profits in U.S. Firms. BAR. Brazilian Administration Review, v. 10, p. 1-17, 2013.

LEE, H.; SMITH, K. G.; GRIMM, C. M.; SCHOMBURG, A. Timing, order and durability of new product advantages with imitation. Strategic Management Journal, v. 21, p. 23-30, 2000.

MAIA, L. G.; BASTOS, A. V. B. Organizational Commitment, Psychological Contract Fulfillment and Job Performance: A Longitudinal Quanti-qualitative Study. Brazilian Administration Review, v. 12, n. 3, p. 250-267, 2015.

MADORRAN, C.; GARCIA, T. Corporate social responsibility and financial performance: the Spanish case. RAE, v. 56, n. 1, p. 20-28, 2016.

MARTINS, H. C.; TERRA, P. R. S. Maturidade do endividamento, desenvolvimento financeiro e instituições legais: análise multinível em empresas latino-americanas. RAUSP, v. 50, n. 3, p. 381-394, 2015.

MITCHELL, T. R.; JAMES, L. R. Building better theory: Time and the specification of when things happen. Academy of Management Review, v. 26, p. 530-548, 2001.

OLIVEIRA, D. DE L.; MAÇADA, A. C. G.; OLIVEIRA, G. D. Valor da Tecnologia da Informação na Firma: Estudo com Empresas Brasileiras. Revista de Administração Contemporânea, v. 19, n. 2, p. 170-192, 2015.

OGASAVARA, M. H. The Role of Experiential Knowledge and Subsequent Investment Decisions on the Profitability of Japanese Companies in Brazil. Brazilian Administration Review, v. 7, p. 59-78, 2010.

ORELLANO, V. I. F.; QUIOTA, S. Análise do retorno dos investimentos socioambientais das empresas brasileiras. RAE, v. 51, n. 5, p. 471-484, 2011.

PALOMBINI, N. V. N.; NAKAMURA, W. T. Key factors in working capital management in the Brazilian market. RAE, v. 52, n. 1, p. 55-69, 2011.

PITARIU, A. H.; PLOYHART, R. E. Explaining Change: Theorizing and Testing Dynamic Mediated Longitudinal Relationships. Journal of Management, v. 36, n. 2, p. 405-429, 2010.

PLOYHARD, R. T.; VANDENBER, R. J. 2010. Longitudinal Research: The Theory, Design, and Analysis of Change. Journal of Management, v. 36, n. 1, p. 94-120, 2010.

RIPAMONTI, A. Corwin-Schultz Bid-ask Spread Estimator in the Brazilian Stock Market. Brazilian Administration Review, v. 13, p. 76-97, 2016.

ROE, R. A. Time in Applied Psychology: The Study of "What Happens" Rather Than "What is". European Psychologist, v. 13, n. 1, p. 37:52, 2008.

SILVA, J. M. A. DA; FAMÁ, R. Evidências de retornos anormais nos processos de IPO na Bovespa no período de 2004 a 2007: um estudo de evento. RAUSP, v. 46, n. 2, p. 178-190, 2011.

SUTTON, R. I.; STAW, B. M. What theory is not. Administrative Science Quarterly, v. 40, p. 371-484, 1995.

TIMMONS, A. C.; PREACHER, K. J. The Importance of Temporal Design: How do measurement intervals affect the accuracy and efficiency of parameter estimates in longitudinal research. Multivariate Behavioral Research, v. 50, p. $41-55,2015$.

VESCO, D. G. D.; BEUREN, I. M. Do the Board of Directors Composition and the Board Interlocking Influence on Performance? Brazilian Administration Review, v. 13, n. 2, p. 1-26, 2016.

WIDLOK, T. Agency, Time, and Causality. Frontiers in Psychology, v. 5, p. 1264, 2014. Doi: 10.3389/fpsyg.2014.01264. 